O

\title{
Adult-onset hereditary pulmonary alveolar proteinosis caused by a single-base deletion in CSF2RB
}

Takeshi Tanaka, ${ }^{1}$ Natsuki Motoi, ${ }^{2}$ Yoshiko Tsuchihashi, ${ }^{1}$ Ryushi Tazawa, ${ }^{2}$ Chinatsu Kaneko, ${ }^{2}$ Takahito Nei, ${ }^{2}$ Toshiyuki Yamamoto, ${ }^{3}$ Tomayoshi Hayashi, ${ }^{4}$ Tsutomu Tagawa, ${ }^{5}$ Takeshi Nagayasu, ${ }^{5}$ Futoshi Kuribayashi, ${ }^{6}$ Koya Ariyoshi, ${ }^{1}$ Koh Nakata, ${ }^{2}$ Konosuke Morimoto ${ }^{1}$

\begin{abstract}
- Additional figures and tables are published online only. To view these files please visit the journal online (http://jmg.bmj. com).

${ }^{1}$ Department of Clinical Medicine, Institute of Tropical Medicine, Nagasaki University, Nagasaki, Japan

${ }^{2}$ Bioscience Medical Research Center, Niigata University Medical \& Dental Hospital Niigata, Japan

${ }^{3}$ Tokyo Women's Medical University, Institute for Integrated Medical Sciences, Tokyo, Japan

${ }^{4}$ Department of Pathology, Nagasaki University Hospital, Nagasaki, Japan

${ }^{5}$ Division of Surgical Oncology, Department of Surgery, Nagasaki University Graduate School of Biomedical Sciences, Nagasaki, Japan ${ }^{6}$ Department of Biochemistry, Kawasaki Medical School, Okayama, Japan
\end{abstract}

\section{Correspondence to} Dr Koh Nakata, Bioscience Medical Research Center, Niigata University Medical \& Dental Hospital, 1-754, Asashimachi-dori, Chuo-ku, Niigata 951-8520, Japan; radical@med.niigata-u.ac.jp

$\mathrm{T} \mathrm{T}$ and $\mathrm{N} \mathrm{M}$ contributed equally to this article.

Received 30 June 2010 Revised 29 July 2010 Accepted 1 October 2010 Published Online First 12 November 2010

\section{ABSTRACT \\ Background Disruption of granulocyte/macrophage colony-stimulating factor (GM-CSF) signalling causes pulmonary alveolar proteinosis (PAP). Rarely, genetic defects in neonatal or infant-onset PAP have been identified in CSF2RA. However, no report has clearly identified any function-associated genetic defect in CSF2RB.}

Methods and results The patient was diagnosed with PAP at the age of 36 and developed respiratory failure. She was negative for GM-CSF autoantibody and had no underlying disease. Signalling and genetic defects in GM-CSF receptor were screened. GM-CSF-stimulated STAT5 phosphorylation was not observed and GM-CSF$\mathrm{R} \beta \mathrm{c}$ expression was defective in the patient's blood cells. Genetic screening revealed a homozygous, single-base deletion at nt 631 in exon 6 of CSF2RB on chromosome 22, which caused reductions in GM-CSF dependent signalling and function. Both parents, who were second cousins, showed no pulmonary symptoms, and had normal GM-CSF-signalling, but had a CSF2RB allele with the identical deletion, indicating that the mutant allele may give rise to PAP in an autosomal recessive manner. Conclusions This is the first report identifying a genetic defect in CSF2RB that causes deficiency of GM-CSF-R $\beta c$ expression and impaired signalling downstream. These results suggested that GM-CSF signalling was compensated by other signalling pathways, leading to adult-onset PAP.

\section{INTRODUCTION}

Pulmonary alveolar proteinosis (PAP) is a rare lung disease in which lipoproteinaceous material is accumulated in the alveoli and terminal bronchioles, resulting in the development of respiratory failure. PAP is clinically divided into three distinct forms: autoimmune, secondary and hereditary PAP. ${ }^{1}$ Autoimmune PAP is associated with the disruption of granulocyte/macrophage colony-stimulating factor (GM-CSF) signalling caused by high levels of GM-CSF autoantibodies in the lung. ${ }^{3}$ Very rarely, there are unclassified cases in which neither GM-CSF autoantibodies nor underlying diseases are confirmed. ${ }^{4}$ Of significance is the fact that mutant mice deficient in GM-CSF or its receptor spontaneously develop PAP $^{6}$ and some hereditary cases exhibit defects in GM-CSF receptor (GM-CSF-R) expression. $^{78}$

GM-CSF- $R$ is composed of the binding $\alpha$ chain and the common $\beta$ chain, which is also utilised by interleukin-3 (IL-3) and IL-5. Binding of GM-CSF initiates the Jak2, STAT5 and PI3K/Akt pathways.

Recently, two groups reported mutations in the coding region of the GM-CSF-R $\alpha$ gene (CSF2RA) in hereditary PAP cases, which is located in the pseudoautosomal region of PAR1 on the $\mathrm{X}$ and $\mathrm{Y}$ chromosomes. $^{78}$

Dirksen et al reported GM-CSF-R $\beta c$ deficiency in four patients with PAP diagnosed during the neonatal to infant period. In one patient, a point mutation in CSF $2 R B$ resulted in an amino acid change at codon 602 from proline to threonine. However, this mutation is now known as a single nucleotide polymorphism in normal subjects ${ }^{10}$ (NCBI SNP Database ID rs1801122; http://www. genecards.org/), and thus, no report has clearly identified gene defects in CSF2RB disrupting GM-CSF signalling. Here, we describe the first adult-onset case with a single-base deletion in exon 6 of CSF2RB, resulting in severe reductions in GM-CSF dependent signalling and function in the blood cells.

\section{METHODS}

\section{Subjects}

This study was approved by the institutional review boards of Nagasaki University and Niigata University. Written informed consent was obtained from the patient, her parents and all control volunteers. Blood mononuclear cells were collected from the subjects. Genetic counselling was performed for the patient and her parents before and after genetic analyses.

\section{GM-CSF autoantibody measurement}

GM-CSF autoantibody concentrations in the serum and bronchoalveolar lavage fluid (BALF) were measured by ELISA as described previously. ${ }^{4}$

Isolation of blood mononuclear cells and monocytes Heparinised blood was obtained and blood mononuclear cells were prepared by density gradient centrifugation using Lymphoprep (Axis-Shield, Oslo, Norway). To isolate CD14 positive monocytes from blood mononuclear cells, human CD14 microbeads (MACS; Miltenyi Biotec, Auburn, California, USA) were applied for magnetic sorting

\section{Differentiation of monocytes to macrophages in vitro}

Peripheral blood monocytes from a control subject and the patient were cultured in a 24 -well culture 
plate (BD Falcon, Franklin Lakes, New Jersey, USA) at $1 \times 10^{5}$ cells per well with $10 \mathrm{ng} / \mathrm{ml}$ of recombinant human GM-CSF (Leukine, Berlex, Seatle, Washignton, USA) in Dulbecco's Modified Eagle Medium (DMEM) containing 10\% heat-inactivated fetal bovine serum (FBS), $2 \mathrm{mM}$ L-glutamine, $100 \mathrm{mg} / \mathrm{ml}$ streptomycin and $100 \mathrm{U} / \mathrm{ml}$ penicillin in a humidified atmosphere of $5 \% \mathrm{CO}_{2}$ at $37^{\circ} \mathrm{C}$. Morphological changes were evaluated after 5-14 days under phase contrast microscopy.

\section{GM-CSF clearance assay}

To assess receptor-mediated binding and uptake of exogenous GM-CSF, blood mononuclear cells from the patient and control volunteer were cultured in 24 -well culture plates at $1 \times 10^{6}$ cells per well in DMEM containing 10\% heat-inactivated FBS, $2 \mathrm{mM}$ L-glutamine, $100 \mathrm{mg} / \mathrm{ml}$ streptomycin and $100 \mathrm{U} / \mathrm{ml}$ penicillin in a humidified atmosphere of $5 \% \mathrm{CO}_{2}$ at $37^{\circ} \mathrm{C}$. Recombinant human GM-CSF was added at a concentration of $100 \mathrm{pg} / \mathrm{ml}$. Subsequently, GM-CSF concentration in the supernatant of each well was measured by ELISA (R\&D Systems, Minneapolis, Minnesota, USA), as previously described. ${ }^{7}$

\section{Immunoblotting}

Blood mononuclear cells were cultured on 24-well culture plates at $5 \times 10^{5}$ cells per well. Cells were incubated with GM-CSF $(0-1000 \mathrm{ng} / \mathrm{ml})$ or IL-3 $(0-10 \mathrm{ng} / \mathrm{ml})$ for $15 \mathrm{~min}$. Lysed protein extracts were separated by sodium dodecylsulphate polyacrylamide gel electrophoresis, transferred to a polyvinylidene fluoride (PVDF) membrane, and assessed by standard western blotting procedures, as previously described. ${ }^{\text {. Primary antibodies }}$ used for detection included anti-human GM-CSF-R $\beta c$ (Santa Cruz Biotechnology, Santa Cruz, California, USA), anti-human GM-CSF-R $\alpha$, anti-human STAT5 (Santa Cruz Biotechnology), and anti-human phosphor-STAT5 (Millipore, Billerica, Massachusetts, USA) antibodies. Peroxidase labelled anti-rabbit IgG antibody (Sigma-Aldrich, St Louis, Missouri, USA) was used as second antibody and visualised with ECL plus (GE Healthcare, Waukesha, Wisconsin, USA). To enhance the signal, immunoreactions enhancer solution (Can Get Signal, TOYOBO, Osaka, Japan) was used according to the manufacturer's instructions. Actin was measured as a loading control for each sample using anti-actin antibody (Santa Cruz Biotechnology).

\section{Flow cytometry}

CD11b expression of neutrophils was analysed by flow cytometry (FACSCalibur, Beckton Dickinson, Franklin Lakes, New Jersey, USA) as previously described. ${ }^{7}$ Briefly, heparinised whole blood was collected and incubated in the absence or presence of $10 \mathrm{mg} / \mathrm{ml}$ human GM-CSF. Blood was incubated with a phycoerythrin (PE) conjugated mouse anti-human CD11b (BD Pharmingen, Franklin Lakes, New Jersey, USA) and a fluorescein isothiocyanate (FITC) conjugated mouse anti-human CD16 (BD Pharmingen). Each triplicate sample was analysed. Expression of GM-CSF receptors was analysed using anti-human CD116 (GM-CSF-R $\alpha$ ) (eBioscience, San Diego, California, USA) and anti-human CD131 (common $\beta$ ) (eBioscience).

\section{Reverse transcription PCR}

Total RNA was extracted from blood mononuclear cells using RNA Easy Plus Mini Kit (OIAGEN, Hilden, Germany) and was reverse transcribed with random hexamer primers using the SuperScript III First-Strand Synthesis System for reverse transcription (RT)-PCR (Invitrogen, Carlsbad, California, USA). The cDNAs were subjected to semi-quantitative RT-PCR analysis using PrimeSTAR GXL DNA polymerase (TaKaRa Bio,
Otsu, Japan) with CSF2RA-specific primer (supplementary table 2).

\section{Nucleotide sequencing}

PCR products were purified using OIAquick DNA extraction kit (OIAGEN) and subjected to nucleotide sequencing using BigDye Terminator V3.1 cycle sequencing kit (Applied Biosystems, Foster City, California, USA) and CSF2RB-specific primer (supplementary table 2).

\section{Array-CGH}

Array-comparative genomic hybridisation (aCGH) analysis was performed using the Agilent 105A Whole Human Genome Oligo Microarray kit according to the manufacturer's protocol (Agilent Technologies, Palo Alto, California, USA). Genomic DNA was extracted from peripheral blood using the OIAquick DNA extraction kit (OIAGEN).

\section{RESULTS}

In 2000, a 36-year-old woman, previously healthy, with normal chest radiograph at a health check-up 1 year earlier, gradually developed dyspnoea on exertion. She was diagnosed as having PAP by typical findings on chest radiography (figure 1A), bronchoalveolar lavage (BAL), and lung histopathological examination (figure $1 \mathrm{~B}$ ). She had undergone whole lung lavage (WLL) five times within 4 years and had subsequently started home oxygen therapy. In 2005, the arterial blood gas analysis on room air showed $\mathrm{a} \mathrm{PaO}_{2}$ of $44.8 \mathrm{~mm} \mathrm{Hg}$. Her clinical parameters and course are summarised in supplementary figure 1 and supplementary table 1, respectively. Bone marrow cells showed mild hypocellularity with normal cell differentials and a normal karyotype of bone marrow cells. GM-CSF autoantibodies were not detected in either the serum or BALF (supplementary table 1).

We decided to screen for GM-CSF signalling abnormalities because GM-CSF concentration was extremely high in her serum $(124.8 \mathrm{pg} / \mathrm{ml}) .^{7}$ The patient's monocytes incubated with GM-CSF failed to differentiate into macrophages with a viability of less than $50 \%$ within 5 days (figure 1C). The baseline expression of CD11b, a complement receptor of neutrophils, was up-regulated in the patient compared with the control, which was augmented by incubation with GM-CSF in the control but not in the patient (figure 1D). Clearance of GM-CSF by the patient's peripheral blood mononuclear cells (PBMCs) was impaired (figure 1E). We then analysed signal activated by GM-CSF in PBMCs. When PBMCs were stimulated with increasing concentrations of GM-CSF $(0-1000 \mathrm{ng} / \mathrm{ml})$, phosphorylated STAT5 was observed in the healthy control but not in the patient's PBMCs (figure 2A). Similarly, phosphorylated STAT5 was not observed in the IL-3-stimulated PBMCs, suggesting defective signalling mediated by GM-CSF-R $\beta c$ (figure $2 \mathrm{~B}$ ). Using flow cytometry, the expression of GM-CSF-R $\alpha$ on patient monocytes was comparable to the control; however, expression of GM-CSF-R $\beta c$ was not detected (figure $2 \mathrm{C}$ ). These results were confirmed at mRNA level by RT-PCR (figure 2D) and immunoblotting (figure $2 \mathrm{E}$ ).

CDNA of CSF2RA reverse transcribed from the mRNA of the patient PBMCs had no mutations or deletions (data not shown). PCR amplicons for 14 exons of CSF2RB from patient PBMCs appeared similar to the control in size and expression (figure $2 \mathrm{~F}$ ). Screening of nucleotide sequences from the PCR amplicon for each exon revealed a homozygous single-base deletion at nt 631 in exon 6 (accession number: AB573019) (figure 2G), causing a frame shift that gave rise to a stop codon at nt 789 (supplementary figure $2 \mathrm{~A}$ ). This deletion has not been reported in the 
Figure 1 Pulmonary alveolar proteinosis (PAP) diagnosis and granulocyte/macrophage colonystimulating factor (GM-CSF) functional analysis on leucocytes. (A) Chest highresolution CT demonstrating diffuse homogenous ground-grass opacity and crazy-paving appearance. (B) Section of lung biopsy specimen stained with $\mathrm{H \& E}$, demonstrating accumulation of amorphous eosinophilic materials in alveolar space (total magnification $40 \times$ ). Bar: $1.0 \mathrm{~mm}$. (C) Phase contrast microscopic images of monocytes from the patient (right) or a control (left) incubated with $10 \mathrm{ng} / \mathrm{ml}$ of GM-CSF for 5 days. Results shown are representative of independent experiments. (D) Flow cytometry analyses for the expression of CD11b on neutrophils from a control (upper) and the patient (lower) incubated with (right) or without (left) $10 \mathrm{mg} / \mathrm{ml} \mathrm{GM-CSF}$. Results shown are representative of independent experiments. (E) Time course for absorption of exogenous GMCSF by peripheral blood mononuclear cells (PBMCs) from the patient or a control subject. Cells were incubated as described in methods section. Results are expressed as a percentage of initial GM-CSF concentration. Data are expressed as mean \pm SE. GM-CSF in the culture supernatant was unchanged up to $48 \mathrm{~h}$ in the patient PBMCs, whereas it was rapidly decreased in the control, indicating that GM-CSF was not absorbed by the patient's cells.

SNPs database (UCSC Genome Browser Bioinformatics (http:// genome.ucsc.edu/) and the JSNP Japanese Single Nucleotide Polymorphism Database (http://snp.ims.u-tokyo.ac.jp/index. html)). The same deletion was recognised at the same codon in the patient's buccal mucosa, indicating that this change occurred in the germ line (data not shown). Further evaluation using array-comparative genomic hybridisation $(\mathrm{aCGH})$ demonstrated no interstitial deletion encompassing the region of $C S F 2 R B$ (supplementary figure $2 \mathrm{~B}$ ). Taken together with the expression defect on PBMCs, mRNA harbouring a homozygous premature termination codon in the patient's CSF2RB gene may be degraded by nonsense-mediated mRNA decay system. ${ }^{11}$

The nucleotide sequence of exon 6 in CSF2RB from the PBMCs of both parents demonstrated heterozygous deletion at the same codon as the patient (figure 2G); however, GM-CSF stimulated STAT5 phosphorylation was normal (figure $2 \mathrm{H}$ ). In this regard, Suzuki et al reported a case with CSF2RA mutation whose parents showed heterozygous mutations but normal STAT5 phosphorylation under GM-CSF stimulation. ${ }^{7} 12$ Although CSF $2 R B$ and CSF2RA are different genes, it may support our finding that a heterozygous mutation in GM-CSF receptor genes does not cause the signalling defect. Furthermore, according to the functional analysis in $\beta c(-/-)$ mice, proliferating capacity of bone marrow cells under GM-CSF stimulation was intact in heterozygous $(+/-)$ mice compared with that in wild-type $(+/+)$ mice. $^{6}$ Although both parents were free from any pulmonary disease, they had been second cousins with a history of three miscarriages between them. Therefore, the homozygous single-base deletion in CSF2RB was probably inherited from both parents in an autosomal recessive manner.

\section{DISCUSSION}

This is the first report identifying a gene defect in CSF2RB causing GM-CSF-R $\beta c$ deficiency and a signalling defect which caused adult-onset hereditary PAP. In a previous report, flow cytometry evaluation showed that four out of eight cases of hereditary PAP had complete GM-CSF-R $\beta$ c deficiency, whereas the remaining four cases showed reduced expression. ${ }^{9}$ All of these diagnoses were made within the first 17 months of infancy when all patients became symptomatic. Four patients with a complete defect in GM-CSF-R $\beta c$ expression had severe respiratory failure and required oxygen therapy. A point mutation within CSF2RB was found in one patient, but was later recognised to be present in $6 \%$ of 184 multinational normal subjects as mentioned above.

Targeting deletion of GM-CSF-R $\beta \mathrm{c}$ in mice has been shown to give rise to the development of PAP between 6 and 14 weeks after birth, which can be reversed by bone marrow transplantation and haematopoietic reconstitution by $8-12$ weeks after the transplant. ${ }^{13}$ However, the defect in surfactant clearance was significantly milder in CSF2RB targeted mice than in GM-CSF deficient mice. ${ }^{14}$ In this regard, Ding et al hypothesised that multiple intracellular signalling pathways are being activated by GM-CSF binding to the GM-CSF-R $\alpha$ alone. ${ }^{15}$

Thus, we speculate that the onset of PAP in the present case might have been delayed by the signals from extremely elevated GM-CSF in the lung, which was conveyed through GM-CSF-R $\alpha$. Alternatively, some factors other than GM-CSF in the lung may have compensated for the function of alveolar macrophages. This was supported by the presence of mild leukocytosis on admission and remarkably increased M-CSF levels in BALF, in 
Figure 2 Effect of granulocyte/ macrophage colony-stimulating factor (GM-CSF) on functions of leucocytes, and expression and genetic analyses of GM-CSF-R $\beta$ c. (A) GM-CSF stimulated phosphorylation of STAT5 in peripheral blood mononuclear cells (PBMCs). PBMCs from the patient or a control subject were incubated with GM-CSF $(0-1000 \mathrm{ng} / \mathrm{ml})$. Phosphorylated STAT5 (pSTAT5) was detected by western blotting. As positive controls, total STAT5 (STAT5) and actin were detected. Results shown are representative of independent experiments. (B) IL-3 stimulated phosphorylation of STAT5 in PBMCs was evaluated by western blotting. PBMCs from the patient or a control subject were incubated with IL-3 $(0-10 \mathrm{ng} / \mathrm{ml})$. Results shown are representative of independent experiments. (C) Flow cytometry analysis for the expression of GM-CSF$R \alpha$ and $\beta c$ on monocytes from a control (upper) and the patient (lower). (D) RTPCR analysis of mRNA in PBMCs from a control (left) or the patient (right) using primers specific for cDNA of CSF2RA (lower) or CSF2RB (upper). (E) Detection of GM-CSF-R $\beta c$ (upper), $\alpha$ (middle) and actin (lower) on PBMCs by western blotting from a control (left) and the patient (right) using anti-GM-CSF-R $\beta \mathrm{c}$, $a$ and actin antibodies, respectively. No band was observed in the patient (right lane) at the position of band for GMCSF-R $\beta$ which was detected in the control (left lane), whereas bands for GM-CSF-R $\alpha$ and actin were clearly detected in both the patient and the control. Enhanced chemiluminescence was used for the detection of the signal. Results shown are representative of independent experiments. (F)

Comparison of PCR amplicons for the 14 exons (exon1-14) from genomic DNA of CSF2RB between a control and the patient.

A

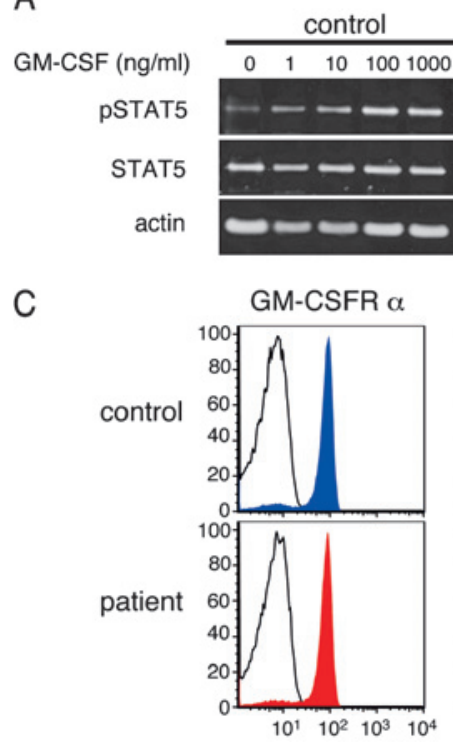

E

G

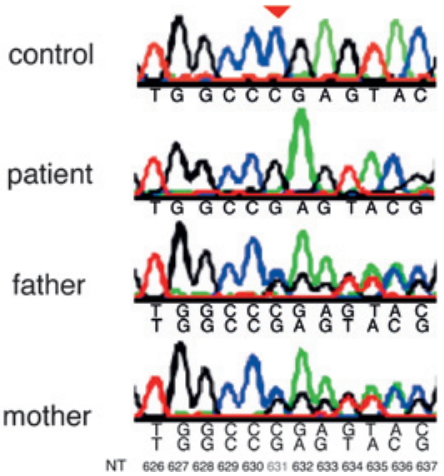

$\mathrm{B}$ IL-3(10 ng/ml) $\frac{\text { control }}{\frac{+}{30} \frac{-}{30}} \frac{\text { patient }}{\frac{t}{01530} \frac{-}{30}}$ stimulation(min)

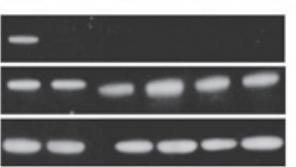

pSTAT5

STAT5

actin

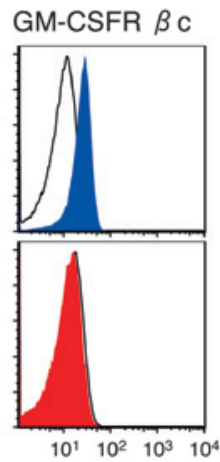

D

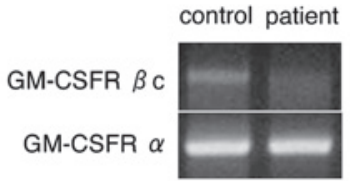

$\mathrm{F}$
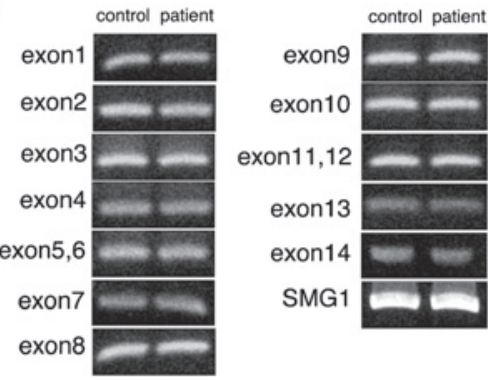

$\mathrm{H}$

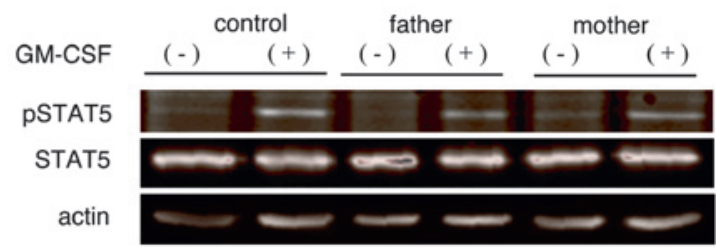

(G) Nucleotide sequence of CSF2RB from a control, the patient and patient's parents, encoding nt 626-637(numbered relative to the initiation codon; GenBank accession NM 000395). (H) GM-CSF stimulated phosphorylation of STAT5 in PBMCs. PBMCs from a control, the patient's father and mother were incubated with or without GM-CSF $(10 \mathrm{ng} / \mathrm{ml})$. pSTAT5 was detected by western blotting. As positive controls, total STAT5 and actin were detected. Results shown are representative of independent experiments.

the absence of infectious disease. In this regard, the late onset of PAP in the present case was distinctive and unique among $\beta c$ deficiency.

Taking the previous report into consideration, ${ }^{7}$ the detection of phosphorylated STAT5 in blood mononuclear cells stimulated with GM-CSF and/or IL-3 is likely to be a useful and convincing method of screening for defects in GM-CSF-R $\alpha$ signalling. This case had some similarities of phenotype of PAP and molecular biomarkers (serum GM-CSF, serum SP-D, GM-CSF in BAL, M-CSF in BAL, CD11b SI, STAT5 phosphorylation, and GM-CSF clearance) with CSF2RA mutations cases. ${ }^{12}$ Increased GM-CSF levels in BALF and serum are important markers since autoimmune PAP is typically characterised by neutralisation of GM-CSF in the lung. Thus, an elevated serum free GM-CSF level is useful in distinguishing between autoimmune and GM-CSF receptor dysfunction PAP. ${ }^{16}$

Bone marrow transplantation would represent the most effective treatment for our case. In this regard, Dirksen et al reported that allogeneic bone marrow transplantation for a patient with acute myelogenous leukaemia successfully led to remission of secondary PAP. ${ }^{17}$ Fukuno et al described a case of PAP secondary to myelodysplastic syndrome that resolved after successful cord blood transplantation. ${ }^{18}$ Replacement of impaired alveolar macrophages with stem cell derived normal alveolar macrophages might correct the defect seen in PAP. However, the safety and efficacy of bone marrow or cord blood transplantation for this patient should be further evaluated. $^{19}$ 
Acknowledgements The authors thank Drs Bruce Trapnell, Takuji Suzuki and Jun Tohyama for helpful advice and discussions.

Funding This work was supported in part by grants from the Japanese Ministry of Education and Science, the Ministry of Health, Labor and Welfare of Japan (H15-trans-014), and the Japanese Society for the Promotion of Science (200390230, 20659130).

Competing interests None.

Patient consent Obtained.

Ethics approval This study was conducted with the approval of the institutional review boards of Nagasaki University and Niigata University.

Provenance and peer review Not commissioned; externally peer reviewed.

\section{REFERENCES}

1. Trapnell BC, Whitsett JA, Nakata K. Pulmonary alveolar proteinosis. N Eng/ J Med 2003:349:2527-39.

2. Kitamura T, Tanaka N, Watanabe J, Uchida K, Kanegasaki S, Yamada Y, Nakata K. Idiopathic pulmonary alveolar proteinosis as an autoimmune disease with neutralizing antibody against granulocyte/macrophage colony-stimulating factor. J Exp Med 1999;190:875-80

3. Uchida K, Nakata K, Trapnell BC, Terakawa T, Hamano E, Mikami A, Matsushita I, Seymour JF, Oh-eda M, Ishige I, Eishi Y, Kitamura T, Yamada Y, Hanaoka K, Keicho N. High-affinity autoantibodies specifically eliminate granulocyte-macrophage colonystimulating factor activity in the lungs of patients with idiopathic pulmonary alveolar proteinosis. Blood 2004;103:1089-98.

4. Inoue Y, Trapnell BC, Tazawa R, Arai T, Takeda T, Hizawa N, Kasahara Y, Tatsumi K, Hojo M, Ichiwata T, Tanaka N, Yamaguchi E, Eda R, Oishi K, Tsuchihashi Y, Kaneko C, Nukiwa T, Sakatani M, Krischer JP, Nakata K. Characteristics of a large cohort of patients with autoimmune pulmonary alveolar proteinosis in Japan. Am J Respir Crit Care Med 2008:177:752-62

5. Dranoff G, Crawford AD, Sadelain M, Ream B, Rashid A, Bronson RT, Dickersin GR, Bachurski CJ, Mark EL, Whitsett JA, Mulligan RC. Involvement of granulocytemacrophage colony-stimulating factor in pulmonary homeostasis. Science 1994;264:713-16

6. Robb L, Drinkwater CC, Metcalf D, Li R, Köntgen F, Nicola NA, Begley CG. Hematopoietic and lung abnormalities in mice with a null mutation of the common beta subunit of the receptors for granulocyte-macrophage colony-stimulating factor and interleukins 3 and 5. Proc Natl Acad Sci U S A 1995:92:9565-9.

7. Suzuki T, Sakagami T, Rubin BK, Nogee LM, Wood RE, Zimmerman SL, Smolarek T, Dishop MK, Wert SE, Whitsett JA, Grabowski G, Carey BC, Stevens C, van der Loo JC, Trapnell BC. Familial pulmonary alveolar proteinosis caused by mutations in CSF2RA. J Exp Med 2008;205:2703-10.
8. Martinez-Moczygemba M, Doan ML, Elidemir O, Fan LL, Cheung SW, Lei JT, Moore JP, Tavana G, Lewis LR, Zhu Y, Muzny DM, Gibbs RA, Huston DP. Pulmonary alveolar proteinosis caused by deletion of the GM-CSFRalpha gene in the $\mathrm{X}$ chromosome pseudoautosomal region 1. J Exp Med 2008;205:2711-16

9. Dirksen U, Nishinakamura R, Groneck P, Hattenhorst U, Nogee LM, Murray R, Burdach S. Human pulmonary alveolar proteinosis associated with a defect in GM-CSF/IL-3/IL-5 receptor common beta chain expression. J Clin Invest 1997:100:2211-17

10. Carey B, Trapnell BC. The molecular basis of pulmonary alveolar proteinosis. Clin Immunol 2010;135:223-35.

11. Chang YF, Imam JS, Wilkinson MF. The nonsense-mediated decay RNA surveillance pathway. Annu Rev Biochem 2007;76:51-74.

12. Suzuki T, Sakagami T, Young LR, Carey BC, Wood RE, Luisetti M, Wert SE, Rubin BK, Kevill K, Chalk C. Whitsett JA, Stevens C, Nogee LM, Campo I, Trapnell BC. Hereditary pulmonary alveolar proteinosis: pathogenesis, presentation, diagnosis, and therapy. Am J Respir Crit Care Med. Published Online First: 2010 July 9. doi:10.1164/ rccm.201002-02710C

13. Nishinakamura R, Wiler R, Dirksen U, Morikawa Y, Arai K, Miyajima A, Burdach S Murray R. The pulmonary alveolar proteinosis in granulocyte macrophage colonystimulating factor/interleukins $3 / 5$ beta c receptor-deficient mice is reversed by bone marrow transplantation. J Exp Med 1996;183:2657-62.

14. Reed JA, Ikegami M, Robb L, Begley CG, Ross G, Whitsett JA. Distinct changes in pulmonary surfactant homeostasis in common b-chain- and GM-CSF-deficient mice. Am J Physiol Lung Cell Mol Physiol 2000:278:L1164-71.

15. Ding DX, Rivas Cl, Heaney ML, Raines MA, Vera JC, Golde DW. The alpha subunit of the human granulocyte-macrophage colony-stimulating factor receptor signals for glucose transport via a phosphorylation-independent pathway. Proc Natl Acad Sci USA 1994;91:2537-41.

16. Carraway MS, Ghio AJ, Carter JD, Piantadosi CA. Detection of granulocytemacrophage colony-stimulating factor in patients with pulmonary alveolar proteinosis Am J Respir Crit Care Med 2000;161:1294-9.

17. Dirksen U, Hattenhorst U, Schneider P, Schroten H, Göbel U, Böcking A, Müller KM Murray R, Burdach S. Defective expression of granulocyte-macrophage colony stimulating factor/interleukin-3/interleukin-5 receptor common beta chain in children with acute myeloid leukemia associated with respiratory failure. Blood 1998;92:1097-103

18. Fukuno K, Tomonari A, Tsukada N, Takahashi S, Ooi J, Konuma T, Uchiyama M, Fuji T, Endo T, Iwamoto A, Oyaizu N, Nakata K, Moriwaki H, Tojo A, Asano S Successful cord blood transplantation for myelodysplastic syndrome resulting in resolution of pulmonary alveolar proteinosis. Bone Marrow Transplant 2006;38:581-2.

19. Parimon T, Madtes DK, Au DH, Clark JG, Chien JW. Pretransplant lung function, respiratory failure, and mortality after stem cell transplantation. Am J Respir Crit Care Med 2005;172:384-90 